Results 143 paramedics undertook the survey and 858 vignettes were completed. There was clear agreement between paramedics for transport decisions $(\kappa=0.63)$ and for admission prediction $(\kappa=0.86)$. Overall accuracy was 0.69 (95\% CI: 0.66 to 0.73 ). Paramedics were better at 'ruling in' the ED with sensitivity of 0.89 (95\% CI: 0.86 to 0.92 ). The specificity of 'ruling out' the ED was 0.51 (95\% CI: 0.46 to 0.56 ). Text comments were focused on patient safety and risk aversion.

Conclusion Paramedics make accurate conveyance decisions but are more likely to over-convey than under-convey, meaning that whilst decisions are safe they are not always appropriate. Some risk-averse decisions were made due to patient and professional safety reasons. It is important that paramedics feel supported by the service to make non-conveyance decisions. Reducing over-conveyance is a potential method of reducing ED demand.

Conflict of interest None

Funding None

\section{USE OF THE SBARR IN HANDOVERS FROM THE AMBULANCE SERVICE TO THE EMERGENCY DEPARTMENT}

${ }^{1} \mathrm{G}$ Geuzebroek* ${ }^{*}{ }^{2} \mathrm{E}$ Zwaan, ${ }^{1,2} \mathrm{~S}$ de Vries, ${ }^{3} \mathrm{MDF}$ Rhebergen, ${ }^{4} \mathrm{~J}$ Berdowski, ${ }^{1} \mathrm{MP}$ Gorzeman, ${ }^{5} \mathrm{~F}$ Holleman. ${ }^{1}$ Ambulance Amsterdam; ${ }^{2}$ OLVG - Amsterdam; ${ }^{3}$ Netwerk Acute Zorg Noordwest; ${ }^{4}$ SpoedZorgNet AMC; ${ }^{5}$ AMC - Amsterdam, The Netherlands

\subsection{6/10.1136/bmjopen-2018-EMS.63}

Aim One of the main causes of adverse events in the healthcare chain is communication failure. Information loss from handovers could lead to a prolonged stay in the Emergency Department (ED) and reduced patient safety. Objectives in this study were to evaluate the accurate use of the SBARR and to evaluate the influence of the (appropriate) use of the SBARR on the agreement between the probable diagnosis after handover and the final diagnosis and time of stay at the ED.

Method Data on handovers were collected through observations in 4 hospitals in Amsterdam, from February until May 2017. Subsequently multiple interventions to improve the use of SBARR were implemented (education, newsletters, e-learning, posters and notebooks). This was followed by a second observation period from September until November 2017.

Results A total of 990 handovers was observed. 45 Handovers were excluded because these were transfers patients from other hospitals or patients refused to participate. Of the remaining 945 handovers, $14(1,5 \%)$ used only one element of the SBARR and $242(25,6 \%)$ of handovers used 2 elements. In over half of cases $(567,60 \%) 3$ elements were used. In 107 handovers (11,3\%) 4 elements where used and in 15 handovers $(1,6 \%)$ all 5 elements where used. For the appropriate use of the SBARR, there were 7 handovers which didn't start with the S, $267(28,3 \%)$ which only consisted of the S, $451(47,7)$ with the SB order, 197 (20,8\%) SBA, 17 handovers $(1,8 \%$ SBAR and 6 handovers (0,6\%) SBARR. Other analyses are currently in progress.

Conclusion The use of the SBARR in these observations was suboptimal. With further analysis, we want to study if this also has a important effect on patient oriented outcomes, like length of stay in the emergency department.

Conflict of interest None

Funding This project is funded by ZonMw (The Netherlands Organisation for Health Research and Development).

\section{4 \\ DIAGNOSTIC PATTERN OF PATIENTS WITH ACUTE CHEST PAIN AND SYMPTOMS OF PATIENTS WITH MYOCARDIAL INFARCTION}

${ }^{1}$ D Davodian, ${ }^{1} \mathrm{TA}$ Lindskou, 1,2 $\mathrm{MB}$ Søvsø, 1,3 TM Larsen, ${ }^{1} \mathrm{TA}$ Kløjgaard, ${ }^{1} \mathrm{HO}$ Holdgaard*,

${ }^{1,2,4}$ EF Christensen. ${ }^{1}$ Center for Prehospital and Emergency Research, Department of Clinical Medicine, Aalborg University, Aalborg, Denmark; '2Emergency Medical Services, North Denmark Region, Aalborg, Denmark; ${ }^{3}$ Unit of Business Intelligence, North Denmark Region, Aalborg, Denmark; ${ }^{4}$ Department of Anaesthesiology and Intensive Care, Emergency Clinic, Aalborg University Hospital, Aalborg, Denmark

\subsection{6/10.1136/bmjopen-2018-EMS.64}

Aim Ischaemic heart diseases including acute myocardial infarction (MI) are regarded as one of the causes of chest pain. The study aimed to investigate the diagnostic pattern and aetiology of prehospital patients, either presenting with chest pain as main symptom or receiving MI as diagnosis at discharge.

Method The study was a retrospective, register-based cohort study describing prehospital patients either presenting with chest pain or diagnosed with MI in the North Denmark Region. Patients were included if they were transferred to a hospital in the region after prior contact with emergency medical services during 2011-2014.

Results Out of a total 71870 prehospital patients, there were 10679 patients with chest pain (14.9\% of all). The diagnoses were diseases of the circulatory system $(35.3 \%, 9.6 \%$ with $\mathrm{MI})$, the respiratory system $(5.2 \%)$, the digestive system (3.8\%), and 'nonspecific diagnoses' (46.1\%). 1365 patients (1.9\% of all prehospital patients) were diagnosed with MI. These mainly presented with chest pain (75.0\%), unclear problems (9.1\%), and difficulty in breathing (7.3\%). When comparing MI-patients presenting with chest pain and patients without chest pain (1,024 vs 341 patients), more were men (71.8\% vs $59.9 \%)$, had lower mortality (4.9\% vs $27.7 \%)$, and a comorbidity score of $0(64.0 \%$ vs $44.3 \%)$. The sensitivity of chest pain as test for MI was $75.0 \%$ and the specificity was $86.3 \%$.

Conclusion The majority of patients with chest pain had other diagnoses than circulatory diseases, and 90.4\% did not have MI. However, most MI-patients presented with chest pain and these had better outcomes than the ones without chest pain.

Conflict of interest None

Funding The professorship of EFC is supported by a grant administered by Aalborg University from the Danish philanthropic foundation TrygFonden. The foundation had no influence on the research.

\section{CAN ROUTINE BLOOD GAS SCREENING IDENTIFY PATIENTS WITH UNSUSPECTED ACID-BASE CONDITIONS AND LEAD TO OPTIMISED TRIAGE GROUP ALLOCATION?}

${ }^{1,2,3} \mathrm{M}$ Lumholdt*, ${ }^{1,2} \mathrm{KA}$ Damgaard, ${ }^{4} \mathrm{EF}$ Christensen, ${ }^{3,4} \mathrm{P}$ Leutscher. ${ }^{1}$ Department of Anesthesiology, North Denmark Regional Hospital, Denmark; 'Emergency department, North Denmark Regional Hospital, Denmark; ${ }^{3}$ Centre for Clinical Research, North Denmark Regional Hospital, Denmark; ${ }^{4}$ Clinical Institute, Aalborg University, Denmark

\subsection{6/10.1136/bmjopen-2018-EMS.65}

Aim Arterial blood gas is essential in the assessment of acutely ill patients. However, triage tools do not identify all highacuity patients. ${ }^{1}$ The v-TAC method can convert venous blood gas (VBG) values to arterial values (aVBG), which facilitate rapid blood gas sampling. ${ }^{2}$ The aim of this study is to 